\title{
Therapeutic Options in Myelodysplastic Syndromes Following Hypomethylating Agent Failure
}

\author{
Authors: \\ Abigail Belasen, ${ }^{1}$ Shyamala C. Navada ${ }^{2}$ \\ 1. Department of Medicine, Icahn School of Medicine, New York City, New York, USA \\ 2. Tisch Cancer Institute, Division of Hematology/Oncology, Icahn School of Medicine, \\ New York City, New York, USA \\ *Correspondence to shyamala.navada@mssm.edu
}

Disclosure: $\quad$ The authors have declared no conflicts of interest.

Received: $\quad 04.12 .19$

Accepted: $\quad 06.01 .20$

Keywords: $\quad$ Hypomethylating agent failure, hypomethylating agents (HMA), myelodysplastic syndromes (MDS), targeted therapies, treatment.

Citation: $\quad$ EMJ Hematol US. 2020;1[1]:52-64.

\section{Abstract}

Hypomethylating agents (HMA) azacitidine and decitabine are standard of care for the treatment of myelodysplastic syndromes (MDS). Although HMA have revolutionised the treatment of MDS, only approximately half of patients respond to these agents with variable duration of effect, known as primary and secondary HMA failure, respectively. Therapeutic options following HMA failure remain limited; however, growing understanding of the pathogenesis underlying MDS has resulted in the development of multiple targeted therapies showing varying degrees of success in clinical trials. Drugs that target molecular alterations (such as abnormal histone regulation, IDH mutations, and spliceosome gene mutations), abnormal signalling pathways (such as the multikinase inhibitor rigosertib), cellular apoptosis (such as the Bcl2 inhibitor venetoclax), and immune checkpoint inhibition are under development. Agents recently approved for use in higher-risk acute myeloid leukaemia, such as FLT3-inhibitors and CPX-351, are also being studied in MDS. Several more agents, including two first-in-class agents, a novel immune regulator targeting CD47, and pevonedistat, a NEDD8-activating enzyme inhibitor, are under investigation. In the absence of established therapeutic approaches following HMA failure, decisions in therapy should be based on the type of HMA resistance as well as the patient's clinical and molecular characteristics. As targeted therapies continue to be developed, a comprehensive re-evaluation of the patient including the mutational profile at the time of HMA failure may reveal new treatment options. Here, emerging therapeutic approaches to HMA failure in MDS are reviewed.

\section{INTRODUCTION}

Myelodysplastic syndromes (MDS) are a heterogeneous group of disorders defined by ineffective haematopoiesis and clonal instability with risk of transformation to acute myeloid leukaemia (AML). ${ }^{1}$ Goals of therapy are to reduce

the symptom burden from cytopenias and decrease the risk of progression of disease. Only three drugs have been approved by the U.S. Food and Drug Administration (FDA) for use in MDS: lenalidomide, an orally administered immunomodulatory drug; and two parenterally administered nucleoside analogue hypomethylating agents (HMA), azacitidine 
and decitabine. HMA have been the standard of care for patients with MDS for over a decade.,3 Azacitidine, first approved in 2004, received expanded approval in 2008 for patients with higher-risk MDS based on the large, randomised Phase III AZA-001 trial which showed a median overall survival (OS) of 24.5 months compared to 15.0 months in patients receiving supportive care. ${ }^{2}$ Decitabine was approved in 2006 based on Phase III study results showing an overall response rate (ORR) of $17 \%$ compared to $0 \%$ of patients receiving supportive care. ${ }^{3}$ However, only azacitidine has been shown to prolong survival in MDS. While the European Medicines Agency (EMA) has approved HMA for International Prognostic Scoring System (IPSS; Table 1) ${ }^{4}$ intermediate and higher-risk MDS, some countries such as the USA also utilise these agents in lower-risk patients. A revised IPSS (IPSS-R; Table 2) was developed in 2012; however, established therapies were approved using the original IPSS system. ${ }^{5}$ Recent data suggests a benefit to early intervention in lower-risk patients. ${ }^{6}$ Although HMA prolong survival, the response is transient and as many as half of patients will not respond to these agents. Moreover, there are different types of HMA failure, including absence of response, or progression of disease or failure following an initial response, termed primary and secondary failure, respectively. Regardless of the nature of failure, incapability to respond denotes a poor prognosis with models suggesting median OS of 4.5 months and 11.0 months in higher and lowerrisk patients, respectively. ${ }^{7}$ Therapeutic options following HMA failure remain limited with no standard of care approach. Fortunately, growing understanding of the pathogenesis of MDS and AML have led to the development of a variety of targeted therapies with varying degrees of success in clinical trials. Here, the mechanisms of HMA failure and novel therapeutic options in these patients are reviewed (Table 3). ${ }^{8-55}$

\section{DEFINING HYPOMETHYLATING AGENT FAILURE}

Patients should be treated with standard dose of HMA prior to assessing their response to therapy. There are currently two standard of care options: 1) decitabine $20 \mathrm{mg} / \mathrm{m}^{2}$ per day for 5 days; or 2) azacitidine $75 \mathrm{mg} / \mathrm{m}^{2}$ per day for 7 days, each at 4-week intervals for at least 6 cycles. ${ }^{56}$ Interruption of therapy can lead to loss of response or disease progression while re-challenge may not be effective. ${ }^{56}$ Moreover, recommendations from a consensus meeting of international experts recommended continuing HMA, if possible, until overt disease progression to minimise risk of relapse. ${ }^{56}$ Routine follow-up during HMA therapy includes monitoring peripheral blood counts for cytopenias or blasts. Bone marrow evaluation is typically performed every 6 months, or earlier if progression of disease is suspected. ${ }^{56}$ However, even when HMA therapy is optimised, failure may occur in different settings.

Primary response failure, or resistance, to HMA is defined by either the absence of response after at least four to six cycles of therapy demonstrated by stable disease without any of the following: complete remission ( $C R$ ), marrow $C R(m C R)$, partial remission (PR), or haematologic improvement $(\mathrm{HI})$ based on the International Working Group (IWG) criteria, ${ }^{57}$ progression of disease to higher-risk MDS category or to AML, or the discontinuation of therapy as a result of side effects such as hypoplastic marrow or pancytopenia. ${ }^{58}$ Secondary response failure, or resistance, is defined by a loss of response or disease progression following an initial response ( $\mathrm{CR}, \mathrm{mCR}, \mathrm{PR}, \mathrm{HI}$ ) to treatment.

The 'post-HMA model' was recently developed for prognostication following HMA failure. It incorporates the patient's Eastern Cooperative Oncology Group (ECOG) performance status, age at diagnosis, presence of complex cytogenetics, marrow blast percentage $>20$, red cell transfusion dependency, and platelet count $<30,000.59$ Patients in this model are stratified into lower-risk or higher-risk with a median OS of 11.0 and 4.5 months, respectively. ${ }^{59}$ Models like these could assist in clinical trial selection following HMA failure. ${ }^{59}$ Although certain clinical and genetic features may predict favourable responses to $\mathrm{HMA}^{60}$ mechanisms of $\mathrm{HMA}$ resistance remain unclear. $^{61}$ Moreover, while MDS is characterised by DNA and gene-specific hypermethylation, the degree of demethylation following HMA administration is not associated with haematologic response. ${ }^{62,63}$ Research efforts have focussed on identifying modifications in the cellular transport and metabolism of HMA as well as identifying biomarkers associated with HMA response. ${ }^{64}$ 
Table 1: International Prognostic Scoring System (IPSS).

\begin{tabular}{|c|c|c|c|c|c|c|}
\hline & & \multicolumn{5}{|c|}{ IPSS score } \\
\hline & & 0.0 & 0.5 & 1.0 & 1.5 & 2.0 \\
\hline \multirow{3}{*}{ 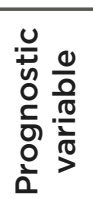 } & Blasts (\%) & $<5$ & $5-10$ & & $11-20$ & $21-30$ \\
\hline & Cytogenetics $^{ \pm}$ & Good & Intermediate & Poor & & \\
\hline & Cytopenias ${ }^{*}$ & O or 1 & 2 or 3 & & & \\
\hline
\end{tabular}

\pm Cytogenetics: good: normal, $-Y$ only, del(5q) only, del(20q) only; Intermediate: abnormalities other than good or poor; Poor: complex $>3$ abnormalities, chromosome 7 abnormalities.

$¥$ Cytopenias: haemoglobin $<10 \mathrm{~g} / \mathrm{dL}$; absolute neutrophil count $<1,500$ cells/ $\mu \mathrm{L}$; platelet count $<100,000 / \mu \mathrm{L}$. IPSS risk score interpretation:

$0.0=$ Low risk

0.5-1.0 = Intermediate-1 risk

1.0-1.5 = Intermediate-2 risk

$\geq 2.5$ = High risk

Adapted from Greenberg et al. ${ }^{4}$

Table 2: Revised International Prognostic Scoring System (IPSS-R).

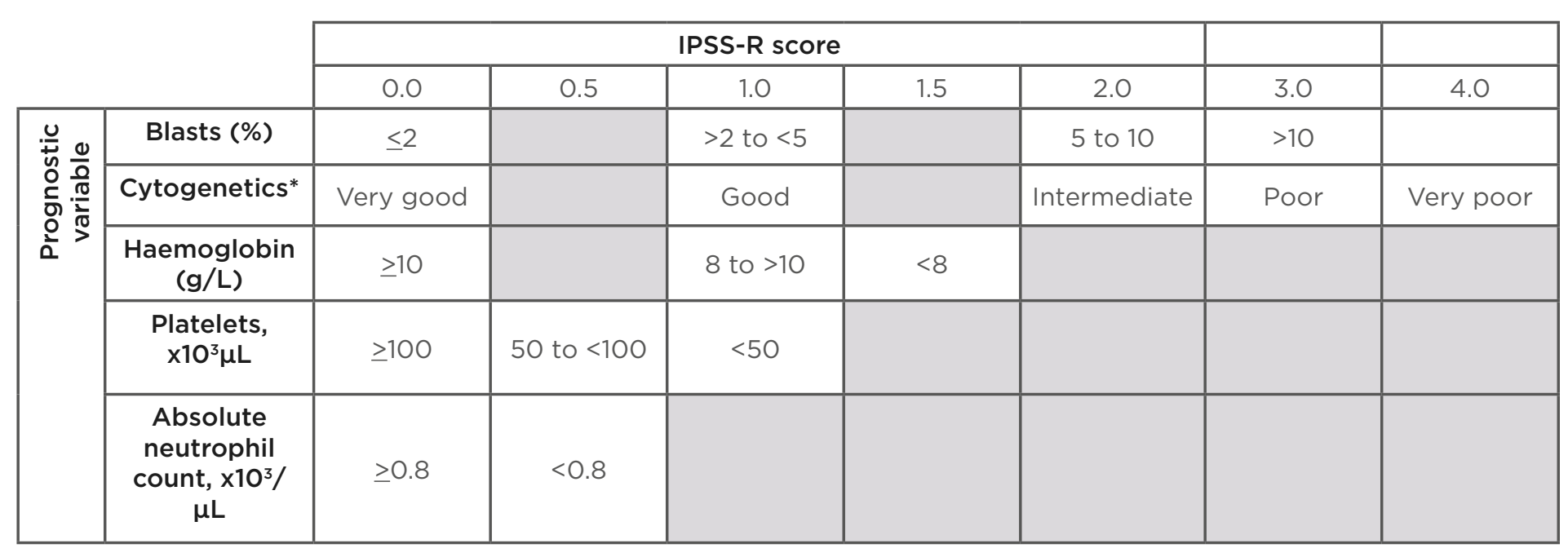

${ }^{*}$ Cytogenetics: very good: del(11q) or -Y; Good: normal, del(20q), del(5q), del(12p), or double including del(5q); Intermediate: +8 , del(7q), $i(17 q),+19$, or any other single or double independent clone; Poor: -7 , inv(3)/t(3q)/de/(3q), double including $-7 /$ del( $7 q$ ), or complex (3 abnormalities); very poor: complex $>3$ abnormalities.

IPSS-R risk score interpretation:

$\leq 1.5=$ Very low risk

$>1.5$ to $3.0=$ Low risk

$>3.0$ to $4.5=$ Intermediate risk

$>4.5$ to $6.0=$ High risk

$>6.0=$ Very high risk

Adapted from Greenberg et al. ${ }^{5}$ 
Table 3: Agents under active investigation in patients with myelodysplastic syndromes (MDS).

\begin{tabular}{|c|c|c|c|c|c|}
\hline Mechanism & Agent & NCT identifier & Phase & Relevant study population & Status \\
\hline \multicolumn{6}{|c|}{ Epigenetic regulators } \\
\hline \multirow{3}{*}{$\begin{array}{l}\text { Hypomethylating } \\
\text { agents }\end{array}$} & \multirow[t]{3}{*}{ Guadecitabine } & NCTO29353618 & II & Int-1 or HR-MDS + HMAf & Recruiting \\
\hline & & NCTO21315979 & II & HR-MDS & \multirow[t]{2}{*}{ Active, not recruiting } \\
\hline & & NCTO290735910 & III & MDS + HMAf & \\
\hline $\begin{array}{l}\text { Histone } \\
\text { deacetylase } \\
\text { inhibitors }\end{array}$ & $\begin{array}{l}\text { Vorinostat, } \\
\text { mocetinostat, } \\
\text { panobinostat, } \\
\text { etc. }\end{array}$ & \multicolumn{4}{|c|}{ No active studies in this population } \\
\hline \multirow{5}{*}{$\begin{array}{l}\text { Mutant IDH1/2 } \\
\text { inhibitors }\end{array}$} & \multirow[t]{2}{*}{ Enasidenib } & NCTO338357511 & II & \multirow[t]{2}{*}{ mIDH2 MDS +/- HMAf } & \multirow[t]{5}{*}{ Recruiting } \\
\hline & & NCTO3744390 ${ }^{12}$ & II & & \\
\hline & \multirow[t]{3}{*}{ Ivosidenib } & NCTO207483913 & 1 & $m / D H 1 \mathrm{r} / \mathrm{r}$ MDS & \\
\hline & & NCTO3471260 14 & $\mathrm{lb} / \mathrm{II}$ & mIDH1 MDS & \\
\hline & & NCTO350340915 & II & $\begin{array}{c}\text { mIDH1: HR-MDS, } \\
\text { treatment-naïve MDS, } \\
\text { ESA-resistant LR-MDS }\end{array}$ & \\
\hline \multirow[t]{3}{*}{ LSD1 inhibitors } & \multirow[t]{2}{*}{ Tranylcypromine } & NCTO227310 $2^{16}$ & I & $\mathrm{r} / \mathrm{r}$ MDS & Active, not recruiting \\
\hline & & NCTO2717884 ${ }^{17}$ & II & Int-/HR-MDS + HMAf & Recruiting \\
\hline & GSK2879552 & \multicolumn{4}{|c|}{ No active studies } \\
\hline \multicolumn{6}{|c|}{ Signal transduction regulators } \\
\hline \multirow{4}{*}{$\begin{array}{l}\text { TGF- } \beta \text { signalling } \\
\text { modulators }\end{array}$} & Galunisertib & \multirow{2}{*}{\multicolumn{4}{|c|}{ No active studies in this population }} \\
\hline & Sotatercept & & & & \\
\hline & \multirow[t]{2}{*}{ Luspatercept } & NCTO2631070 18 & II & $\begin{array}{l}\text { Very low, low, or int-risk } \\
\text { MDS refractory to ESA }\end{array}$ & Active, not recruiting \\
\hline & & NCT03682536 & III & $\begin{array}{l}\text { Very low, low, or int-risk } \\
\text { MDS in ESA-naïve }\end{array}$ & Recruiting \\
\hline TLR inhibitors & $\begin{array}{l}\text { Tomaralimab } \\
\text { (OPN-305) }\end{array}$ & & No ac & tive studies in this populatior & \\
\hline \multirow[t]{5}{*}{$\begin{array}{l}\text { Multi-kinase } \\
\text { inhibitors }\end{array}$} & \multirow[t]{5}{*}{ Rigosertib } & NCTO192658720 & I/II & $\begin{array}{c}\text { Int-1, Int-2 (Int-2), or HR- } \\
\text { MDS }\end{array}$ & \multirow[t]{4}{*}{ Active, not recruiting } \\
\hline & & NCTO1904682 21 & II & LR or Int-1 risk-MDS & \\
\hline & & NCT0192853722 & III & MDS + excess blasts + & \\
\hline & & NCTO1241500 23 & III & HMAf & \\
\hline & & NCTO256244324 & III & $\begin{array}{c}\text { Very high-risk MDS + } \\
\text { HMAf }\end{array}$ & Recruiting \\
\hline \multirow[t]{3}{*}{ FLT-3 inhibitors } & Midostaurin & NCTO0819546 25 & 1 & r/r MDS and AML & Active, not recruiting \\
\hline & Gilteritinib & \multicolumn{4}{|c|}{ No active studies in this population } \\
\hline & Sorafenib & NCTO2728050 26 & II & HR-MDS & Recruiting \\
\hline \multicolumn{6}{|l|}{ Immunotherapy } \\
\hline \multirow[t]{8}{*}{ PD-1 inhibitors } & \multirow[t]{3}{*}{ Nivolumab } & NCTO2530463 27 & II & \multirow[t]{3}{*}{ MDS +/- HMAf } & \multirow[t]{3}{*}{ Recruiting } \\
\hline & & NCTO246465728 & II & & \\
\hline & & NCTO3417154 29 & II & & \\
\hline & Durvalumab & NCTO277590330 & II & Treatment-naïve, HR-MDS & Active, not recruiting \\
\hline & & NCTO2281084 ${ }^{31}$ & II & MDS + HMAf & \\
\hline & Pembrolizumab & NCTO2936752 32 & 1 & MDS +/- HMAf & Recruiting \\
\hline & & NCT0309463733 & II & Int-1 or HR-MDS +/- HMAf & \\
\hline & Atezolizumab & & NCTC & $2935361,{ }^{8}$ see guadecitabine & \\
\hline CTLA-4 inhibitors & Ipilimumab & & $\mathrm{NC}$ & $02530463,{ }^{27}$ see nivolumab & \\
\hline & & NCTO289032934 & I & MDS +/- HMAf & Recruiting \\
\hline
\end{tabular}


Table 3 continued.

\begin{tabular}{|c|c|c|c|c|c|}
\hline Mechanism & Agent & NCT identifier & Phase & Relevant study population & Status \\
\hline $\begin{array}{l}\text { Anti-CD47 } \\
\text { antibody }\end{array}$ & Hu5F9-G4 & NCTO324847935 & I & $\begin{array}{l}r / r \text { and treatment-naïve } \\
\text { MDS }\end{array}$ & Recruiting \\
\hline \multirow{3}{*}{$\begin{array}{l}\text { Bispecific T } \\
\text { cell engaging } \\
\text { antibodies }\end{array}$} & MCLA-117 & \multirow{2}{*}{\multicolumn{4}{|c|}{ No active studies in this population }} \\
\hline & AMG330 & & & & \\
\hline & AMV564 & NCT0351659136 & I & $\begin{array}{c}\text { Int-2 or HR-MDS with } \\
\text { HMAf or standard AML } \\
\text { CTX }\end{array}$ & Active, not recruiting \\
\hline \multicolumn{6}{|c|}{ Cell death regulators } \\
\hline \multirow[t]{7}{*}{$\mathrm{Bcl}-2$ inhibitors } & \multirow[t]{7}{*}{ Venetoclax } & NCTO2966782 37 & I & HR-MDS + HMAf & Active, not recruiting \\
\hline & & NCTO4017546 38 & I & MDS with $\geq 10 \%$ blasts & \multirow[t]{6}{*}{ Recruiting } \\
\hline & & NCTO294229039 & 1 & Treatment-naïve HR-MDS & \\
\hline & & NCTO311364340 & I & HR-MDS & \\
\hline & & NCTO416005241 & $\mathrm{I} / \mathrm{II}$ & HR-MDS +/- HMAf & \\
\hline & & NCTO340419342 & II & HR-MDS + HMAf & \\
\hline & & NCTO211529543 & II & MDS with $\geq 10 \%$ blasts & \\
\hline \multirow{7}{*}{$\begin{array}{l}\text { NEDD } 8 \\
\text { activating } \\
\text { enzyme }\end{array}$} & \multirow[t]{7}{*}{ Pevonedistat } & NCTO377292544 & 1 & HR-MDS + HMAf & \multirow[t]{5}{*}{ Recruiting } \\
\hline & & NCTO381314745 & I & HR-MDS & \\
\hline & & NCTO345985946 & I & MDS +/- HMAf & \\
\hline & & NCTO381400547 & I & HR-MDS + HMAf & \\
\hline & & NCTO323824848 & II & MDS+ HMAf & \\
\hline & & NCTO261077749 & II & HR-MDS & Active, not recruiting \\
\hline & & NCTO326895450 & III & HR-MDS & Recruiting \\
\hline \multicolumn{6}{|l|}{ Other agents } \\
\hline $\begin{array}{l}\text { RNA splicing } \\
\text { modulators }\end{array}$ & H3B-8800 & NCTO2841540 51 & I & $\begin{array}{l}\text { HR-MDS + HMAf, LR-MDS } \\
\text { refractory to ESA }\end{array}$ & Active, not recruiting \\
\hline \multirow[t]{4}{*}{ Cytotoxic agents } & CPX-351 & NCTO201906952 & I & \multirow[t]{2}{*}{ HR-MDS + HMAf } & \multirow[t]{4}{*}{ Recruiting } \\
\hline & & NCTO389626953 & I & & \\
\hline & & NCTO395787654 & II & MDS + HMAf & \\
\hline & & NCT0367253955 & II & HR-MDS + HMAf & \\
\hline
\end{tabular}

CTX: chemotherapy; ESA: erythropoiesis-stimulating agents; HMAf: hypomethylating agent failure; HR: high risk; Int: intermediate; LR: low risk; MDS: myelodysplastic syndromes; $m I D H$ : mutant IDH; r/r: relapsed/refractory;

TLR: toll-like receptor.

\section{CURRENT THERAPEUTIC OPTIONS FOLLOWING HYPOMETHYLATING AGENT FAILURE}

Clinical trials are recommended for patients who fail HMA; however, if not accessible, chemotherapy and haematopoietic stem cell transplant (HSCT) may be used in the appropriate patient.

\section{Cytotoxic Agents and Combinations}

Standard AML-based chemotherapy, classically cytosine arabinoside plus anthracycline $(7+3)$, may be used in patients with higher-risk MDS. However, high-dose chemotherapy is associated with prolonged cytopenias with serious infectious complications, and therefore is better tolerated by younger patients with more favourable cytogenetic profiles., ${ }^{2,64}$ In a larger cohort of 307 patients, of whom 70\% were lower risk, comparison of 3 induction regimens 
(7+3, intermediate versus high dose cytosine arabinoside, or nucleoside analogues) showed similar median OS of 10.8 months, with ORR of $41 \%, 64 \%$, and $34 \%$, respectively. ${ }^{65}$ Low-dose chemotherapy (including low-dose cytarabine, hydroxyurea, mercaptopurine, and low-dose melphalan) has not been shown to be superior to best supportive care (BSC), with median OS of 7.3 months. ${ }^{66}$ However, in a study of predominantly elderly patients with higher-risk MDS after HMA failure, combinations of low-dose cytarabine and clofarabine resulted in a median OS of 10.0 months with 44\% ORR. 67 Of responders, 30\% underwent allogeneic HSCT and 56\% achieved long-term remission.

A liposomal formulation of cytarabine and daunorubicin, known as CPX-351, was approved in August 2017 for therapy-related AML and AML with myelodysplasia-related changes. ${ }^{68}$ Compared to standard 7+3, CPX-351 demonstrated improved OS (hazard ratio [HR]: 0.69; 95\% confidence interval $[\mathrm{Cl}]$ : 0.52-0.90; $\mathrm{p}=0.005$ ), with a median OS of 9.6 months compared to 5.9 months. ${ }^{68}$ Given the success of CPX-351, particularly in AML with myelodysplasia-related changes, researchers are investigating the effects of CPX-351 in elderly patients with MDS and AML following HMA failure in a Phase II clinical trial. ${ }^{52}$

\section{Haematopoietic Stem Cell Transplant}

Allogeneic HSCT is the only potentially curative option for patients with higher-risk MDS. However, given its significant toxicity and mortality, use is limited to younger patients or older patients who have a good performance status and donor. Moreover, studies suggest that patients with MDS who failed to respond to HMA have a higher risk of post-HSCT relapse than patients with positive response to HMA. ${ }^{69}$ In 125 patients treated with HMA, relapse-free survival at 3 years was $23.8 \%$ in patients with primary HMA failure and $42.0 \%$ among patients who previously responded to HMA. ${ }^{69}$

\section{EMERGING THERAPIES FOLLOWING HYPOMETHYLATING AGENT FAILURE}

\section{Novel Approaches to Hypomethylating Agent Therapy}

Despite limited data, sequential use of the alternative HMA as a means of overcoming resistance is not uncommon practice. A few small, mostly retrospective studies have demonstrated between a $19 \%$ and $28 \%$ response rate to decitabine following azacitidine treatment failure ${ }^{70,71}$ and $40 \%$ to azacitidine following decitabine treatment failure. ${ }^{71}$

Empiric addition of other agents to HMA in first-line therapy have been studied, including combinations with lenalidomide and vorinostat, ${ }^{72}$ but have failed to show clinical benefit. Recently, a Phase II study employing a 'pick a winner' approach, investigated several combination therapies in patients with higher-risk MDS with the aim of launching more definitive investigations if a successful combination was found. ${ }^{73}$ Patients were randomly assigned to treatment with azacitidine alone versus azacitidine plus lenalidomide, azacitidine plus valproic acid, or azacitidine plus idarubicin..$^{73}$ None of these combinations were found to be superior to azacitidine alone. ${ }^{73}$ Ongoing combination therapies are being evaluated, including a Phase lb trial investigating azacitidine with venetoclax ${ }^{39}$ and a Phase III trial of azacitidine with pevonedistat. ${ }^{50}$ Novel HMA with oral formulations, longer half-lives, and reduced toxicity are also under development.

\section{Oral Hypomethylating Agents}

Oral azacitidine and decitabine are currently undergoing evaluation in clinical trials. Compared to traditional parenteral formulations, oral formulations allow for delivery of the drug over a longer schedule and provide convenient dosing schedules for patients. Results from Phase I trials in patients with MDS and chronic myelomonocytic leukaemia (CMML) showed treatment with empiric oral azacitidine yielded an ORR of $73 \%$ compared to $35 \%$ in patients who were previously treated with traditional injectable formulations. ${ }^{74}$ Findings from a Phase I study of oral azacitidine in lower-risk patients from this group demonstrated a benefit to extended 
dosing schedules in which azacitidine given as $300 \mathrm{mg}$ once daily for 14 versus 21 day dosing schedule resulted in an ORR of $36 \%$ and $41 \%$, respectively. ${ }^{75}$ A Phase III trial from this group is ongoing. ${ }^{76}$

Cedazuridine, a novel cytidine deaminase inhibitor called ASTX727, increases the bioavailability of oral decitabine by inhibiting its degradation in the gastrointestinal tract and liver. ${ }^{77}$ Phase I studies of oral decitabine in combination with cedazuridine demonstrated similar clinical and biological responses when compared to intravenous decitabine. ${ }^{77}$ Preliminary results from a Phase II study demonstrated clinical benefit in $62 \%$ of patients. ${ }^{78}$ A Phase III study is ongoing. ${ }^{77}$

\section{Guadecitabine}

Guadecitabine is a novel, second-generation HMA resistant to deamination by cytidine deaminase and therefore exhibits longer halflife than decitabine. Two large, Phase II studies were conducted in patients with intermediate-2 or high-risk MDS and CMML either untreated ${ }^{79}$ or following HMA failure. ${ }^{80}$ In the first study, ORR was $61 \%$ with a median OS of 15.0 months at a median follow up of 15.0 months. $^{79}$ The second study, which included patients with HMA failure and treatment-naïve patients, achieved an ORR of $43 \%$ and $51 \%$, respectively, with median OS of 12.0 and 23.1 months. ${ }^{80}$ These results support guadecitabine in first-line therapy. A Phase III trial comparing guadecitabine to standard therapy in patients with MDS and CMML after HMA failure is underway. ${ }^{10}$

\section{OTHER DRUGS TARGETING EPIGENETIC DYSREGULATION}

\section{Histone Deacetylase Inhibitors}

One of the key mechanisms in the epigenetic regulation of gene expression is through histone acetylation and deacetylation. Histone deacetylation results in transcriptional deactivation and ultimately downregulation of gene expression. Transcriptional repression complexes, such as histone deacetylases (HDAC), may downregulate tumour suppressor genes. ${ }^{81}$ Histone deacetylase inhibitors (HDACi) have been assessed for use in MDS alone or in combination with HMA. HDACi may also play a role in apoptosis and induce alterations in the NFKB pathway. ${ }^{81,82}$ Despite robust preclinical data and extensive studies in patients with MDS and $A M L, H D A C i$ have not been shown to improve outcomes in combination therapy with HMA. ${ }^{81,83-85}$

\section{Mutant IDH1 and IDH2 Inhibitors}

Isocitrate dehydrogenases (IDH) are enzymes involved in diverse cellular processes, including histone demethylation and DNA regulation. ${ }^{86}$ Mutations in $I D H 1$ or $I D H 2(m / D H 1 / 2)$ result in DNA and histone hypermethylation impeding haematopoietic progenitor cell differentiation and promoting leukaemogenesis.86 Together, $m / D H 1 / 2$ are among the most common mutations in myeloid malignancies, occurring in approximately $20 \%$ of patients with $\mathrm{AML}$ and $5 \%$ with MDS. ${ }^{87}$ Several $m / D H 1 / 2$ inhibitors are under development as monotherapy or in combination with HMA or chemotherapy. These include two oral agents recently approved by the FDA for use in AML: enasidenib, a $m / D H 2$ inhibitor approved for relapsed/refractory AML with IDH2 mutation; and ivosidenib, a mIDH1 inhibitor approved for AML with IDH1 mutation.

Enasidenib received FDA approval in August 2017 following a Phase I/II study in relapsed/ refractory $A M L$ achieving ORR 40.3\% with CR $19.3 \%{ }^{88}$ A significant survival benefit was seen in patients achieving CR with median OS of 19.7 months versus 9.3 months in relapsed/refractory pateints. ${ }^{88}$ Enasidenib is now being evaluated in patients with MDS after HMA failure, including in two Phase II studies. ${ }^{11,12}$

Similarly, ivosidenib received FDA approval in July 2018 for relapsed/refractory AML based on results from a Phase I study of 125 patients achieving ORR of $42.0 \%$ after 7 months, with CR $22.0 \%$ with median duration of 9.0 months. The median OS was 9 months after a median follow-up of 15.0 months. ${ }^{89}$ Results from an expansion study of 12 patients showed ORR of 91.7\%, with median duration 21.4 months and CR $41.7 \%$ (median duration not estimable).90 Ivosidenib was recently approved as first-line therapy in AML in May 2019. Ivosidenib is being evaluated in a Phase II study in patients with MDS following HMA failure. ${ }^{15}$ 


\section{Lysine Demethylase 1 Inhibitors}

Lysine demethylase 1 (LSD1) regulates gene transcription through the removal of methyl groups from histones and is overexpressed in myeloid malignancies. LSD1 inhibitors (LSD1i) have been shown to promote the differentiation of blast cells in AML, particularly in patients with mutations in KMT2A. ${ }^{91} \mathrm{~A}$ Phase I/II study of LSD1i GSK2879552 in MDS $^{92}$ and Phase I study in AML were both terminated early; however, tranylcypromine, another LSD1i, is currently being evaluated in Phase $1 /$ II studies with results pending. ${ }^{16,17}$

\section{DRUGS TARGETING ABNORMAL SIGNAL TRANSDUCTION}

\section{TGF- $\beta$ Receptor Signalling Modulators}

\begin{abstract}
Abnormal activation of the TGF- $\beta$ receptor signalling pathways has been implicated in the pathogenesis of MDS representing a novel therapeutic target, while suppression of this pathway promotes in vitro haematopoiesis in MDS progenitor cells. ${ }^{93}$
\end{abstract}

Sotatercept, a selective activin receptor ligand that traps GDF11 to restore effective erythropoiesis, was studied in 74 patients, $48 \%$ of whom had HMA failure. $49 \%$ of patients achieved erythroid $\mathrm{HI}$, as did 59\% of patients with HMA failure and $47 \%$ with high transfusion burden. ${ }^{94}$

Galunisertib, a first-in-class oral inhibitor of the TGF- $\beta$ receptor type 1 kinase (ALK5), was recently evaluated as monotherapy in 43 patients with very low, low, or intermediate-risk MDS. ${ }^{95}$ Overall $\mathrm{HI}$ was $24.4 \%(10 / 41)$ and $31.1 \%$ in transfusion-dependent patients $(9 / 28)$ with a median response duration of 3 months. Two patients were previously treated with HMA. Although these agents have yet to be tested in higher-risk patients, findings from these studies suggest treatment with sotatercept and possibly other TGF- $\beta$ receptor signalling modulators may be an option in transfusion-dependent, lower-risk patients following HMA failure.

\section{Toll-like Receptor Inhibitors}

Toll-like receptors (TLR) play a key role in innate immune activation through activation of NF-kB. Overexpression of TLR2 on the MDS cell membrane, which is upregulated by HMA therapy and may be implicated in HMA failure, has been shown to inhibit haematopoietic differentiation in MDS. ${ }^{96}$ Phase $\mathrm{I} / \mathrm{Il}$ studies using tomaralimab (OPN-305), a fully humanised IgG4-K monoclonal antibody against TLR2, were conducted in patients with lower-risk MDS following HMA failure. ${ }^{96}$ Preliminary data suggest an ORR of $50 \%$, supporting a role for tomaralimab in the treatment of lower-risk patients with HMA failure. ${ }^{96}$

\section{Rigosertib Alone and in Combination with Azacitidine}

Rigosertib is a multi-kinase inhibitor of cellular signalling through the targeting of the Rasbinding domain of RAS, PIJK/AKT, and RAF/ PLK, inducing mitotic arrest and apoptosis in neoplastic cells. Rigosertib is currently undergoing investigation as a single agent in certain subtypes of MDS, including patients with higher-risk MDS following HMA failure, as well as in combination with azacitidine. Although intravenous rigosertib was not shown to improve OS compared to BSC in higher risk patients with HMA failure in the Phase III ONTIME trial, a post hoc analysis of very high-risk patients showed median OS significantly improved to 7.6 months in the study group compared to 3.2 months in BSC. ${ }^{97}$ Survival benefit was seen in patients with primary HMA failure, monosomy 7 or trisomy 8 , and who were $<75$ years of age.$^{97} \mathrm{~A}$ second Phase III study is underway, which will further evaluate these patients with very high-risk disease. ${ }^{24}$

An oral formulation of rigosertib is also under investigation. Preclinical data demonstrated a synergistic effect with sequential dosing of rigosertib with azacitidine. ${ }^{98}$ This combination was evaluated in a Phase $\mathrm{I} / \mathrm{Il}$ study of 74 patients with higher-risk MDS..$^{99}$ A dose of $>840 \mathrm{mg}$ per day resulted in ORR $90 \%$ and $54 \%$ in HMA naïve and HMA failure patients, respectively. ${ }^{99} \mathrm{~A}$ Phase III study is anticipated. 


\section{FLT3 Inhibitors}

The FLT3 gene encodes a tyrosine kinase receptor expressed on haematopoietic progenitor cells, which promotes cellular proliferation and differentiation. FLT3 is mutated in approximately $30 \%$ of patients with AML, conferring a poor prognosis with resistance to conventional chemotherapy regimens. ${ }^{100}$ The FLT3 inhibitors midostaurin and gilteritinib have been approved by the FDA in FLT3-mutated AML, the former in combination with $7+3$. Although FLT3 mutations are seen in $<1 \%$ of patients with newly diagnosed MDS, they are found in up to $5 \%$ of patients with MDS transformed to AML. ${ }^{101}$ Phase I/II studies of the FLT3 inhibitors midostaurin and sorafenib, in combination with azacitidine, demonstrated efficacy with ORR of $26 \%$ and $46 \%$, respectively. Although the majority of the study participants had AML, these agents show promise in FLT3-mutated MDS.

\section{IMMUNOTHERAPY}

\section{Immune Checkpoint Inhibitors}

Immune regulatory proteins PD-1/PD-L1 and CTLA-4 downregulate antitumour T-cell responses and promote tumourigenesis. These immune regulatory proteins were upregulated in MDS cells treated with HMA, ${ }^{102}$ which may be linked to HMA failure. The PD-1 inhibitor nivolumab, as well as the CTLA-4 inhibitor ipilimumab, were evaluated in a Phase II study in combination with azacitidine in treatment-naïve MDS or as monotherapy in patients with HMA failure. ${ }^{103}$ In treatment-naïve patients, ORR was 70\% with nivolumab/azacitidine and 62\% with ipilimumab/azacitidine. Median survival was not reached at a median follow up of 20.1 months in treatment-naïve patients treated with ipilimumab/azacitidine, surpassing the effect of azacitidine alone. Further investigations into these agents, including triple combinations, ${ }^{27,104}$ are under investigation.

\section{Anti-CD47 Antibodies}

Hu5F9-G4 (5F9) is a first-in-class anti-CD47 antibody, which targets a key macrophage immune checkpoint resulting in $A M L$ cell phagocytosis. ${ }^{105}$ Azacitidine enhances phagocytic elimination of AML cells when combined with 5F9.106 A Phase I study of 5F9 alone or in combination with azacitidine in patients with relapsed/refractory AML and MDS among other cohorts is under investigation. ${ }^{35}$

\section{Bispecific T-cell Engaging Antibodies}

Bispecific T-cell engaging antibodies link $T$ cells (via the CD3 receptor) with specific antigens on tumour cells to induce tumour cell apoptosis. An increasing number of tumour-specific antigens are under development, including CD33 and CLEC12A, which are frequently expressed on myeloid precursors in AML and MDS. 107,108 Two novel bispecific CD33/CD3 antibodies, AMG330 and AMV564, and a bispecific CLEC12A/CD3 antibody, MCLA-117, are being evaluated in Phase I studies in relapsed/refractory AML. ${ }^{109-111}$ Preliminary results of AMV564 have been encouraging, with reduction in myeloblasts ranging from 13 to $38 \%$ in 6/9 evaluable patients. ${ }^{112}$ AMV564 is also undergoing investigation in intermediate and high-risk MDS in a Phase I study. ${ }^{36}$

\section{RNA splicing modulators}

Dysregulated mRNA splicing has been implicated in tumourigenesis. Genes involved in spliceosome machinery, including SF3B1, SRSF2, U2AF1, and ZRSR2, are frequently mutated in patients with MDS representing novel therapeutic targets. ${ }^{113}$ Based on positive results from preclinical trials, a Phase I study of a novel splicing modulator, $\mathrm{H} 3-\mathrm{B8800}$, is under investigation in MDS patients with HMA failure, CMML, and AML. ${ }^{51}$

\section{DRUGS TARGETING DEREGULATED CELL DEATH PATHWAYS}

\section{$\mathrm{Bcl}-2$ Inhibitors}

$\mathrm{BCl}-2$ is a mitochondrial protein that promotes cellular survival by inhibiting pro-apoptotic pathways. Overexpression of $\mathrm{Bcl}-2$ has been reported in higher-risk MDS leading to resistance of apoptosis ${ }^{114}$ and to azacitidine. ${ }^{115}$ Venetoclax is an orally bioavailable potent inhibitor of $\mathrm{Bcl}$ 2. In November 2018, venetoclax was approved for older adults with newly diagnosed $A M L$ in combination with HMA or low-dose cytarabine who were otherwise not candidates for intensive induction therapy. Interim analysis of a Phase 
Il study ${ }^{42}$ of venetoclax in combination with decitabine achieved a CR/CR with incomplete blood count recovery of $92 \%$ in older patients with newly diagnosed AML, 71\% in secondary $A M L$, and $44 \%$ in patients with relapsed/refractory AML. ${ }^{116}$ Given its success in $A M L$, venetoclax is also being investigated in MDS. A Phase lb study will examine the effect of venetoclax alone or in combination with azacitidine in high-risk patients following HMA failure. ${ }^{37}$

\section{Pevonedistat}

Pevonedistat is a first-in-class inhibitor of the NEDD8 activating enzyme (NAE). NAE is an essential regulator of the degradation of proteins involved in cell cycle progression and cellular stress responses. Preclinical data demonstrated the effect of NAE inhibition in inducing $A M L$ cell death ${ }^{117}$ as well as synergistic effects with azacitidine and decitabine. ${ }^{118}$ A Phase I study of pevonedistat plus azacitidine in treatment-naïve, older patients with AML showed intention-to- treat ORR of $50 \% .^{119}$ This combination is being evaluated in a Phase II study in patents with MDS after HMA failure. ${ }^{48}$ The Phase III PANTHER trial is comparing pevonedistat plus azacitidine to azacitidine alone as first-line therapy in higherrisk MDS, CMML, and low-blast AML. ${ }^{50}$

\section{CONCLUSION}

It is critical to recognise HMA failure in MDS because these patients have poor outcomes. Although there are no standard therapeutic options following HMA failure, several emerging therapies with the goal of improving symptom burden and overall survival are showing promise in clinical studies. These include novel targeted therapies and immune therapies to genes commonly altered in MDS. Therefore, clinical trial enrolment is the preferred option after HMA failure. A comprehensive assessment of the patient's clinical, molecular, and cytogenetic profiles at the time of HMA failure will help guide therapy selection.

\section{References}

1. Nimer SD. Myelodysplastic syndromes. Blood. 2008;111(10):484151.

2. Fenaux $\mathrm{P}$ et al. Efficacy of azacitidine compared with that of conventional care regimens in the treatment of higher-risk myelodysplastic syndromes: a randomised, openlabel, Phase III study. Lancet Oncol. 2009;10(3):223-32.

3. Kantarjian $\mathrm{H}$ et al. Decitabine improves patient outcomes in myelodysplastic syndromes: results of a Phase III randomized study. Cancer. 2006;106(8):1794-803.

4. Greenberg P et al. International scoring system for evaluating prognosis in myelodysplastic syndromes. Blood. 1997;89(6):207988.

5. Greenberg PL et al. Revised international prognostic scoring system for myelodysplastic syndromes. Blood. 2012;120(12):245465.

6. Jabbour E et al. Randomized Phase 2 study of low-dose decitabine vs low-dose azacitidine in lowerrisk MDS and MDS/MPN. Blood. 2017;130(13):1514-22.

7. Prebet $\mathrm{T}$ et al. Predicting outcome of patients with myelodysplastic syndromes after failure of azacitidine: validation of the North American MDS consortium scoring system. Haematologica. 2016;101(10):e427-8.

8. University of Southern California. Guadecitabine and atezolizumab in treating patients with advanced myelodysplastic syndrome or chronic myelomonocytic leukemia that is refractory or relapsed. NCTO2935361. https://clinicaltrials.gov/ct2/show/ NCT02935361.

9. M.D. Anderson Cancer Center. Guadecitabine in treating patients with higher-risk myelodysplastic syndromes. NCTO2131597. https:// clinicaltrials.gov/ct2/show/ NCTO2131597.

10. Astex Pharmaceuticals. Guadecitabine (SGI-110) vs treatment choice in adults with MDS or CMML previously treated with HMAs. NCT02907359. https://clinicaltrials. gov/ct2/show/NCT02907359.

11. M.D. Anderson Cancer Center. Azacitidine and enasidenib in treating patients with $I D H 2$ mutant myelodysplastic syndrome. NCTO3383575. https://clinicaltrials. gov/ct2/show/NCTO3383575.

12. Groupe Francophone des Myelodysplasies. IDH2 (AG 221) inhibitor in patients with IDH2 mutated myelodysplastic syndrome. NCTO3744390. https://clinicaltrials.
gov/ct2/show/NCT03744390.

13. Agios Pharmaceuticals, Inc. Study of orally administered AG-120 in subjects with advanced hematologic malignancies with an IDH1 mutation NCTO2074839. https://clinicaltrials. gov/ct2/show/NCT02074839.

14. M.D. Anderson Cancer Center. Ivosidenib and venetoclax with or without azacitidine in treating participants with IDH1 mutated hematologic malignancies. NCTO3471260. https://clinicaltrials. gov/ct2/show/NCTO3471260.

15. Groupe Francophone des Myelodysplasies. IDH1 (AG 120) inhibitor in patients with $I D H 1$ mutated myelodysplastic syndrome. NCT03503409. https://clinicaltrials. gov/ct2/show/NCT03503409.

16. University of Miami. Phase 1 study of TCP-ATRA for adult patients with AML and MDS (TCP-ATRA). NCTO2273102. https://clinicaltrials gov/ct2/show/NCTO2273102.

17. Michael Luebbert. Study of sensitization of non-M3 AML blasts to ATRA by epigenetic treatment with tranylcypromine (TCP) (TRANSATRA). NCTO2717884. https://clinicaltrials.gov/ct2/show/ NCTO2717884

18. Celgene. A study of luspatercept 
(ACE-536) to treat anemia due to very low, low, or intermediate risk myelodysplastic syndromes (MEDALIST). NCTO2631070. https://clinicaltrials.gov/ct2/show/ NCTO2631070.

19. Celgene. Efficacy and safety study of luspatercept (ACE-536) versus epoetin alfa for the treatment of anemia due to IPSS-R very low, low or intermediate risk myelodysplastic syndromes (MDS) in ESA naïve subjects who require red blood cell transfusions (COMMANDS). NCT03682536. https://clinicaltrials. gov/ct2/show/NCT03682536.

20. Onconova Therapeutics, Inc. Phase Il part 2 expansion of oral rigosertib in combination with azacytidine. NCT01926587. https://clinicaltrials. gov/ct2/show/NCT01926587.

21. Onconova Therapeutics, Inc. Oral rigosertib in low risk MDS patients refractory to ESAs. NCTO1904682. https://clinicaltrials.gov/ct2/show/ NCT01904682.

22. Onconova Therapeutics, Inc Efficacy and safety of IV rigosertib in MDS patients with excess blasts progressing after azacitidine or decitabine. NCTO1928537. https:// clinicaltrials.gov/ct2/show/ NCT01928537.

23. Onconova Therapeutics, Inc. Randomized study of ON 01910.Na in refractory myelodysplastic syndrome patients with excess blasts (ONTIME) NCT01241500. https://clinicaltrials. gov/ct2/show/NCT01241500.

24. Onconova Therapeutics, Inc. Controlled study of rigosertib versus physician's choice of treatment in MDS patients after failure of an HMA (INSPIRE). NCTO2562443. https://clinicaltrials.gov/ct2/show/ NCTO2562443.

25. Richard Stone, MD. RADO01 in combination with PKC412 in patients with relapsed, refractory or poor prognosis AML or MDS. NCTO0819546. https://clinicaltrials. gov/ct2/show/NCT00819546.

26. University of Washington. Filgrastim, cladribine, cytarabine, and mitoxantrone with sorafenib tosylate in treating patients with newlydiagnosed, acute myeloid leukemia or high-risk myelodysplastic syndrome. NCTO2728050. https://clinicaltrials. gov/ct2/show/NCTO2728050.

27. M.D. Anderson Cancer Center. Nivolumab and ipilimumab with 5-azacitidine in patients with myelodysplastic syndromes (MDS). NCTO2530463. https://clinicaltrials. gov/ct2/show/NCTO2530463.

28. M.D. Anderson Cancer Center. Study of idarubicin, cytarabine, and nivolumab in patients with high-risk myelodysplastic syndrome (MDS) and acute myeloid leukemia (AML). NCTO2464657. https://clinicaltrials. gov/ct2/show/NCTO2464657.
29. Masonic Cancer Center, University of Minnesota. Nivolumab and oral cyclophosphamide for R/R AML and high risk MDS. NCT03417154. https://clinicaltrials.gov/ct2/show/ NCT03417154.

30. Celgene. An efficacy and safety study of azacitidine subcutaneous in combination with durvalumab (MEDI4736) in previously untreated subjects with higher-risk myelodysplastic syndromes (MDS) or in elderly subjects with acute myeloid leukemia (AML). NCTO2775903. https://clinicaltrials.gov/ct2/show/ NCTO2775903.

31. Celgene. Safety and efficacy study of CC-486 in subjects with myelodysplastic syndromes. NCT02281084. https://clinicaltrials. gov/ct2/show/NCTO2281084.

32. National Cancer Institute $(\mathrm{NCl})$. Entinostat and pembrolizumab in treating patients with myelodysplastic syndrome after DNMTi therapy failure. NCTO2936752. https://clinicaltrials. gov/ct2/show/NCTO2936752.

33. M.D. Anderson Cancer Center. Azacitidine and pembrolizumab in treating patients with myelodysplastic syndrome. NCT03094637. https://clinicaltrials. gov/ct2/show/NCT03094637.

34. National Cancer Institute ( $\mathrm{NCl}$ ). Ipilimumab and decitabine in treating patients with relapsed or refractory myelodysplastic syndrome or acute myeloid leukemia. NCT02890329. https://clinicaltrials.gov/ct2/show/ NCTO2890329.

35. Forty Seven, Inc. Hu5F9-G4 monotherapy or Hu5F9-G4 in combination with azacitidine in patients with hematological malignancies. NCTO3248479. https://clinicaltrials.gov/ct2/show/ NCT03248479.

36. Amphivena Therapeutics, Inc. A Phase 1 study of AMV564 in patients with intermediate or highrisk myelodysplastic syndromes. NCT03516591. https://clinicaltrials. gov/ct2/show/NCT03516591.

37. AbbVie. A study evaluating venetoclax alone and in combination with azacitidine in subjects with relapsed/refractory myelodysplastic syndromes (MDS). NCTO2966782. https://clinicaltrials.gov/ct2/show/ NCT02966782.

38. Cyclacel Pharmaceuticals, Inc. CYC065 CDK inhibitor and venetoclax study in relapsed/refractory AML or MDS. NCTO4017546. https:// clinicaltrials.gov/ct2/show/ NCT04017546.

39. AbbVie. A study evaluating venetoclax in combination with azacitidine in subjects with treatment-naïve higher-risk myelodysplastic syndromes (MDS). NCTO2942290. https://clinicaltrials. gov/ct2/show/NCTO2942290.
40. Dana-Farber Cancer Institute. SL401 in combination with azacitidine or azacitidine/venetoclax in acute myeloid leukemia (AML) or high-risk myelodysplastic syndrome (MDS). NCT03113643. https://clinicaltrials. gov/ct2/show/NCT03113643.

41. M.D. Anderson Cancer Center. Venetoclax and azacitidine for the treatment of high-risk recurrent or refractory myelodysplastic syndrome. NCT04160052. https://clinicaltrials. gov/ct2/show/NCT04160052.

42. M.D. Anderson Cancer Center. Venetoclax in combination with decitabine in r/r AML. NCTO3404193. https://clinicaltrials.gov/ct2/show/ NCT03404193.

43. M.D. Anderson Cancer Center. Cladribine, idarubicin, cytarabine, and venetoclax in treating patients with acute myeloid leukemia, high-risk myelodysplastic syndrome, or blastic phase chronic myeloid leukemia. NCTO2115295. https://clinicaltrials. gov/ct2/show/NCTO2115295.

44. National Cancer Institute ( $\mathrm{NCl}$ ) Pevonedistat and belinostat in treating patients with relapsed or refractory acute myeloid leukemia or myelodysplastic syndrome. NCT03772925. https://clinicaltrials. gov/ct2/show/NCT03772925

45. National Cancer Institute $(\mathrm{NCl})$ Pevonedistat, azacitidine, fludarabine phosphate, and cytarabine in treating patients with relapsed or refractory acute myeloid leukemia or relapsed high-risk myelodysplastic syndrome NCT03813147. https://clinicaltrials. gov/ct2/show/NCT03813147.

46. Justin Watts. Pevonedistat and low dose cytarabine in adult patients with AML and MDS. NCTO3459859. https://clinicaltrials.gov/ct2/show/ NCTO3459859.

47. Millennium Pharmaceuticals, Inc. A study of pevonedistat in combination with azacitidine in participants with higher-risk myelodysplastic syndromes (HR MDS), chronic myelomonocytic leukemia (CMML), or acute myelogenous leukemia (AML) with severe renal impairment or mild hepatic impairment. NCT03814005. https://clinicaltrials.gov/ct2/show/ NCT03814005

48. Vanderbilt-Ingram Cancer Center. Pevonedistat and azacitidine in MDS or MDS/MPN patients who fail primary therapy with DNA methyl transferase inhibitors. NCTO3238248. https://clinicaltrials.gov/ct2/show/ NCT03238248.

49. Millennium Pharmaceuticals, Inc An efficacy and safety study of pevonedistat plus azacitidine versus single-agent azacitidine in participants with higher-risk myelodysplastic syndromes (HR MDS), chronic myelomonocytic leukemia (CMML) and low-blast acute myelogenous leukemia (AML). 
NCT02610777. https://clinicaltrials. gov/ct2/show/NCTO2610777.

50. Millennium Pharmaceuticals, Inc. Pevonedistat plus azacitidine versus single-agent azacitidine as first-line treatment for participants with higher-risk myelodysplastic syndromes (HR MDS), chronic myelomonocytic leukemia (CMML), or low-blast acute myelogenous leukemia (AML) (PANTHER). NCT03268954. https://clinicaltrials. gov/ct2/show/NCTO3268954.

51. H3 Biomedicine Inc. A Phase 1 study to evaluate H3B-8800 in participants with myelodysplastic syndromes, acute myeloid leukemia, and chronic myelomonocytic leukemia. NCTO2841540. https://clinicaltrials. gov/ct2/show/NCTO2841540.

52. Rondeep Brar. CPX-351 in treating patients with relapsed or refractory acute myeloid leukemia or myelodysplastic syndrome. NCT02019069. https://clinicaltrials. gov/ct2/show/NCTO2019069.

53. M.D. Anderson Cancer Center. CPX-351 in treating patients with relapsed or refractory high risk myelodysplastic syndrome or chronic myelomonocytic leukemia. NCT03896269. https://clinicaltrials. gov/ct2/show/NCT03896269.

54. Case Comprehensive Cancer Center. CPX-351 therapy for MDS after hypomethylating agent failure. NCT03957876. https://clinicaltrials. gov/ct2/show/NCT03957876.

55. M.D. Anderson Cancer Center. Liposome-encapsulated daunorubicin-cytarabine and gemtuzumab ozogamicin in treating patients with relapsed or refractory acute myeloid leukemia (AML) or high risk myelodysplastic syndrome. NCT03672539. https://clinicaltrials. gov/ct2/show/NCT03672539.

56. Santini $\vee$ et al. Minimizing risk of hypomethylating agent failure in patients with higher-risk MDS and practical management recommendations. Leuk Res. 2014;38(12):1381-91.

57. Cheson BD et al. Clinical application and proposal for modification of the International Working Group (IWG) response criteria in myelodysplasia. Blood. 2006:108(2):419-25.

58. Jabbour EJ et al. Outcome of patients with low-risk and intermediate-1risk myelodysplastic syndrome after hypomethylating agent failure: a report on behalf of the MDS Clinical Research Consortium. Cancer. 2015;121(6):876-82.

59. Nazha A et al. The efficacy of current prognostic models in predicting outcome of patients with myelodysplastic syndromes at the time of hypomethylating agent failure. Haematologica. 2016;101(6):e224-7.
60. Ades $L$ et al. Predictive factors of response and survival among chronic myelomonocytic leukemia patients treated with azacitidine. Leuk Res. 2013;37(6):609-13.

61. Wang $\mathrm{H}$ et al. Predictors of clinical responses to hypomethylating agents in acute myeloid leukemia or myelodysplastic syndromes. Ann Hematol. 2018;97(11):2025-38.

62. Fandy TE et al. Early epigenetic changes and DNA damage do not predict clinical response in an overlapping schedule of 5-azacytidine and entinostat in patients with myeloid malignancies. Blood. 2009;114(13):2764-73.

63. Voso MT et al. Why methylation is not a marker predictive of response to hypomethylating agents Haematologica. 2014;99(4):613-9.

64. Valencia A et al. Expression of nucleoside-metabolizing enzymes in myelodysplastic syndromes and modulation of response to azacitidine. Leukemia. 2014;28(3):621-

65. Ball $B$ et al. Evaluation of induction chemotherapies after hypomethylating agent failure in myelodysplastic syndromes and acute myeloid leukemia. Blood Adv. 2018;2(16):2063-71.

66. Prebet $T$ et al. Outcome of high-risk myelodysplastic syndrome after azacitidine treatment failure. J Clin Oncol. 2011;29(24):3322-7.

67. Jabbour $E$ et al. Phase 2 study of low-dose clofarabine plus cytarabine for patients with higher-risk myelodysplastic syndrome who have relapsed or are refractory to hypomethylating agents. Cancer. 2017;123(4):629-37.

68. Krauss AC et al. FDA approval summary: (daunorubicin and cytarabine) liposome for injection for the treatment of adults with high-risk acute myeloid leukemia. Clin Cancer Res. 2019;25(9):2685-90.

69. Festuccia M et al. Hematopoietic cell transplantation in myelodysplastic syndromes after treatment with hypomethylating agents. Biol Blood Marrow Transplant. 2017;23(9):150914

70. Borthakur $\mathrm{G}$ et al. Activity of decitabine in patients with myelodysplastic syndrome previously treated with azacitidine. Leuk Iymphoma. 2008;49(4):690-5.

71. Apuri S et al. Evidence for selective benefit of sequential treatment with hypomethylating agents in patients with myelodysplastic syndrome. Clin Lymphoma Myeloma Leuk. 2017;17(4):211-4.

72. Sekeres MA et al. Randomized Phase II study of azacitidine alone or in combination with lenalidomide or with vorinostat in higher-risk myelodysplastic syndromes and chronic myelomonocytic leukemia: North American intergroup study SWOG S1117. J Clin Oncol. 2017:35(24):2745-53.

73. Ades $L$ et al. A randomized Phase II study of azacitidine (AZA) alone or with lenalidomide (LEN), valproic acid (VPA) or idarubicin (IDA) in higher-risk MDS: Gfm's 'pick a winner' trial. Blood. 2018;132(1):467.

74. Garcia-Manero $\mathrm{G}$ et al. Phase I study of oral azacitidine in myelodysplastic syndromes, chronic myelomonocytic leukemia, and acute myeloid leukemia. J Clin Oncol. 2011;29(18):2521-7.

75. Garcia-Manero G et al. Efficacy and safety of extended dosing schedules of CC-486 (oral azacitidine) in patients with lowerrisk myelodysplastic syndromes. Leukemia. 2016;30(4):889-96.

76. Celgene. The efficacy and safety of oral azacitidine plus best supportive care versus placebo and best supportive care in subjects with red blood cel (RBC) transfusion-dependent anemia and thrombocytopenia due to international prognostic scoring system (IPSS) low risk myelodysplastic syndrome (MDS) NCT01566695. https://clinicaltrials. gov/ct2/show/NCT01566695.

77. Savona MR et al. An oral fixed-dose combination of decitabine and cedazuridine in myelodysplastic syndromes: a multicentre, open-label, dose-escalation, Phase 1 study. Lancet Haematol. 2019;6(4):e194-203.

78. Garcia-Manero $\mathrm{G}$ et al. A Phase 2 dose-confirmation study of oral ASTX727, a combination of oral decitabine with a cytidine deaminase inhibitor (CDAl) cedazuridine (E7727), in subjects with myelodysplastic syndromes (MDS). Blood. 2017;130(Suppl 1):4274.

79. Garcia-Manero $\mathrm{G}$ et al. Final report of a Phase II study of guadecitabine (SGI-110) in patients (pts) with previously untreated myelodysplastic syndrome (MDS). Blood. 2018;132(Suppl 1):232.

80. Garcia-Manero $\mathrm{G}$ et al Guadecitabine (SGI-110) in patients with intermediate or high-risk myelodysplastic syndromes: Phase 2 results from a multicentre, open-label, randomised, Phase 1/2 trial. Lancet Haematol. 2019;6(6):e317-27.

81. Griffiths EA, Gore SD. DNA methyltransferase and histone deacetylase inhibitors in the treatment of myelodysplastic syndromes. Semin Hematol. 2008;45(1):23-30.

82. Johnstone RW, Licht JD. Histone deacetylase inhibitors in cancer therapy: is transcription the primary target? Cancer cell. 2003;4(1):13-8.

83. Prebet $\mathrm{T}$ et al. Prolonged 
administration of azacitidine with or without entinostat for myelodysplastic syndrome and acute myeloid leukemia with myelodysplasia-related changes: results of the US Leukemia Intergroup trial E1905. J Clin Oncol. 2014;32(12):1242-8.

84. Prebet $\mathrm{T}$ et al. Addition of suberoylanilide hydroxamic acid (Vorinostat) to azacitidine for patients with higher risk myelodysplastic syndromes and azacitidine failure: a Phase II add-on study from the Groupe Francophone des Myelodysplasies. Br J Haematol. 2018;180(5):735-7.

85. Garcia-Manero G et al. Phase 2, randomized, double-blind study of pracinostat in combination with azacitidine in patients with untreated, higher-risk myelodysplastic syndromes. Cancer. 2017;123(6):9941002.

86. Medeiros BC et al. Isocitrate dehydrogenase mutations in myeloid malignancies. Leukemia. 2017;31(2):272-81.

87. Figueroa ME et al. Leukemic IDH1 and $I D H 2$ mutations result in a hypermethylation phenotype, disrupt TET2 function, and impair hematopoietic differentiation. Cancer cell. 2010;18(6):553-67.

88. Stein EM et al. Enasidenib in mutant IDH2 relapsed or refractory acute myeloid leukemia. Blood. 2017;130(6):722-31.

89. DiNardo CD et al. Durable remissions with ivosidenib in IDH1-mutated relapsed or refractory AML. N Engl J Med. 2018;378:2386-98.

90. Dinardo $\mathrm{C}$ et al. Ivosidenib (AG120) induced durable remissions and transfusion independence in patients with IDH1-mutant relapsed or refractory myelodysplastic syndrome: results from a Phase 1 dose escalation and expansion study. Blood. 2018;132(Suppl 1):1812.

91. Fiskus $\mathrm{W}$ et al. Highly effective combination of LSD1 (KDM1A) antagonist and pan-histone deacetylase inhibitor against human AML cells. Leukemia. 2014;28(11):2155 64.

92. GlaxoSmithKline. Safety, clinical activity, pharmacokinetics (PK) and pharmacodynamics study of GSK2879552, alone or with azacitidine, in subjects with high risk myelodysplastic syndromes (MDS). NCTO2929498. https://clinicaltrials. gov/ct2/show/NCTO2929498.

93. Zhou $L$ et al. Inhibition of the TGFbeta receptor I kinase promotes hematopoiesis in MDS. Blood. 2008;112(8):3434-43

94. Komrokji R et al. Sotatercept with long-term extension for the treatment of anaemia in patients with lowerrisk myelodysplastic syndromes: a
Phase 2, dose-ranging trial. Lancet Haematol. 2018;5(2):e63-72.

95. Santini $\vee$ et al. Phase II study of the ALK5 inhibitor galunisertib in very low-, low-, and intermediate-risk myelodysplastic syndromes. Clin Cancer Res. 2019. doi: 10.1158/10780432.CCR-19-1338.

96. Garcia-Manero $\mathrm{G}$ et al. A clinical study of tomaralimab (OPN-305), a toll-like receptor 2 (TLR-2) antibody, in heavily pre-treated transfusion dependent patients with lower risk myelodysplastic syndromes (MDS) that have received and failed on prior hypomethylating agent (HMA) therapy. Blood. 2018;132(Suppl 1):798.

97. Garcia-Manero $\mathrm{G}$ et al. Rigosertib versus best supportive care for patients with high-risk myelodysplastic syndromes after failure of hypomethylating drugs (ONTIME): a randomised, controlled, Phase 3 trial. Lancet Oncol. 2016;17(4):496-508.

98. Skidan I et al. Toxicology of a novel small molecule ON1910Na on human bone marrow and leukemic cells in vitro. Proc Amer Assoc Cancer Res. 2006;66(8).

99. Navada $\mathrm{S}$ et al. Phase 2 expansion study of oral rigosertib combined with azacitidine (AZA) in patients (pts) with higher-risk (HR) myelodysplastic syndromes (MDS): efficacy and safety results in HMA treatment naïve \& relapsed (rel)/ refractory (ref) patients. Blood. 2018;132(Suppl 1):230.

100. Levis M, Small D. FLT3: ITDoes matter in leukemia. Leukemia. 2003;17(9):1738-52.

101. Badar T et al. Detectable FLT3-ITD or RAS mutation at the time of transformation from MDS to AML predicts for very poor outcomes. Leuk Res. 2015;39(12):1367-74.

102. Yang $\mathrm{H}$ et al. Expression of PDL1, PD-L2, PD-1 and CTLA4 in myelodysplastic syndromes is enhanced by treatment with hypomethylating agents. Leukemia. 2014;28(6):1280-8

103. Garcia-Manero G et al. A Phase II study of nivolumab or ipilimumab with or without azacitidine for patients with myelodysplastic syndrome (MDS). Blood. 2018;132(Suppl 1):465.

104. Garcia-Manero G et al. Double immune checkpoint inhibitor blockade with nivolumab and ipilimumab with or without azacitidine in patients with myelodysplastic syndrome (MDS). Blood. 2018;132(Suppl 1):1831.

105. Sallman DA et al. The first-in-class anti-CD47 antibody Hu5F9-G4 is active and well tolerated alone or with azacitidine in AML and MDS patients: initial Phase $1 \mathrm{~b}$ results. J Clin Oncol. 2019;37:7009.
106. Feng $D$ et al. Combination treatment with 5F9 and azacitidine enhances phagocytic elimination of acute myeloid leukemia. Blood. 2018;132(Suppl 1):2729.

107. Sanford D et al. CD33 is frequently expressed in cases of myelodysplastic syndrome and chronic myelomonocytic leukemia with elevated blast count. Leuk Lymphoma. 2016;57(8):1965-8.

108. Toft-Petersen $M$ et al. Unravelling the relevance of CLEC12A as a cancer stem cell marker in myelodysplastic syndrome. $\mathrm{Br} \mathrm{J}$ Haematol. 2016;175(3):393-401.

109. Amgen. A Phase 1 study of AMG 330 in subjects with relapsed/ refractory acute myeloid leukemia. NCTO2520427. https://clinicaltrials. gov/ct2/show/NCTO2520427.

110. Amphivena Therapeutics, Inc. Study of AMV564 in patients with AML. NCT03144245. https://clinicaltrials. gov/ct2/show/NCT03144245.

111. Merus N.V. MCLA-117 in acute myelogenous leukemia. NCTO3038230. https://clinicaltrials. gov/ct2/show/NCT03038230.

112. Westervelt $P$ et al. Phase 1 first-inhuman trial of AMV564, a bivalent bispecific (2×2) CD33/CD3 T-cell engager, in patients with relapsed/ refractory acute myeloid leukemia (AML). Presentation 834. ASH Annual Meeting, 9 December, 2019.

113. Yoshida $\mathrm{K}$ et al. Frequent pathway mutations of splicing machinery in myelodysplasia. Nature. 2011;478(7367):64-9.

114. Jilg $\mathrm{S}$ et al. Blockade of BCL-2 proteins efficiently induces apoptosis in progenitor cells of high-risk myelodysplastic syndromes patients. Leukemia. 2016;30(1):112-23.

115. Cluzeau T et al. BCL2L10 is a predictive factor for resistance to azacitidine in MDS and AML patients. Oncotarget. 2012;3(4):490-501.

116. Maiti A et al. Interim analysis of Phase II study of venetoclax with 10-day decitabine (dec10-ven) in acute myeloid leukemia and myelodysplastic syndrome. Blood. 2018;132(Suppl 1):286.

117. Swords RT et al. Inhibition of NEDD8activating enzyme: a novel approach for the treatment of acute myeloid leukemia. Blood. 2010;115(18):3796800.

118. Smith PG et al. Azacitidine/decitabine synergism with the NEDD8-activating enzyme inhibitor MLN4924 in pre-clinical AML models. Blood. 2011;118(21):578.

119. Swords RT et al. Pevonedistat, a firstin-class NEDD8-activating enzyme inhibitor, combined with azacitidine in patients with AML. Blood. 2018;131(13):1415-24. 